\title{
PNAD COVID-19: um novo e poderoso instrumento para Vigilância em Saúde no Brasil
}

\author{
PNAD COVID-19: A powerful new tool for Public Health \\ Surveillance in Brazil
}

Gerson Oliveira Penna (http://orcid.org/0000-0001-8967-536X) 1,2

José Agenor Alvares da Silva (http://orcid.org/0000-0001-7966-0447) ${ }^{2}$

José Cerbino Neto (http://orcid.org/0000-0001-9254-917X) ${ }^{3}$

José Gomes Temporão (https://orcid.org/0000-0001-7162-2905) ${ }^{4}$

Luiz Felipe Pinto (http://orcid.org/0000-0002-9888-606X) ${ }^{5}$

\footnotetext{
${ }^{1}$ Núcleo de Medicina Tropical, Universidade de Brasília. Campus Universitário Darcy Ribeiro, Asa Norte. 70904-900 Brasília DF Brasil. gerson.penna@fiocruz.br ${ }^{2}$ Escola Fiocruz de Governo, Fundação Oswaldo Cruz Brasília. Brasília DF Brasil. ${ }^{3}$ Takemi Program in International Health, Harvard T. H. Chan School of Public Health, Harvard University. Boston MA USA.

${ }^{4}$ Centro de Estudos Estratégicos, Fundação Oswaldo Cruz. Rio de Janeiro RJ Brasil. ${ }^{5}$ Departamento de Medicina em Atenção Primária, Faculdade de Medicina, Universidade Federal do Rio de Janeiro. Rio de Janeiro RJ Brasil.
}

\begin{abstract}
On February 3, 2020, the Brazilian Ministry of Health declared a state of emergency in public health of national relevance due to the pandemic caused by the new coronavirus SARSCoV-2. As a result, IBGE postponed the 2020 Demographic Census and started to formulate a COVID-19 PNAD. The survey included a total sample of 349 thousand people in about 200 thousand households. Of the total Brazilian resident population, the IBGE estimated in May/2020 that 24.0 million (11.4\%) had at least one of the flulike syndrome symptoms. Of this contingent, 20.2 million (84.3\% of all symptomatic patients) did not seek health care. The innovations brought to health surveillance and the IBGE's pioneering spirit show that it is possible, in a continental country that has been experiencing several local epidemics at different times in its territory, that other countries also develop similar household surveys, with weekly data collection (referred to epidemiological weeks) by telephone in an innovative and timely manner. The COVID-19 PNAD also brought new technology to the Institute, reviving its role as an external evaluator of the Unified Health System (SUS).
\end{abstract}

Key words Household survey, COVID-19, Brazil
Resumo O Ministério da Saúde declarou em 03 de fevereiro de 2020 estado de emergência em saúde pública de importância nacional em decorrência da pandemia pelo novo coronavírus SARSCoV-2. Com isso, o IBGE adiou a realização do Censo Demográfico de 2020 e passou a formular uma PNAD COVID-19. O inquérito contou com uma amostra total de 349 mil pessoas em cerca de 200 mil domicílios. Do total da população-residente brasileira, o IBGE estimou em maio/2020 que 24,0 milhões (11,4\%) tiveram pelo menos um dos sintomas de sindrome gripal (SG). Desse contingente, 20,2 milhões ( $84,3 \%$ do total dos sintomáticos) não procuraram unidade de saúde. As inovações trazidas para a vigilância em saúde e o pioneirismo do IBGE demonstram ser possivel, em um país continental e que vem experimentando diversas epidemias locais em momentos diferentes em seu território, que outros países também desenvolvam inquéritos domiciliares semelhantes, com coleta de dados semanal (referida às semanas epidemiológicas) por telefone de forma inovadora e tempestiva. A PNAD COVID-19 trouxe ainda uma nova tecnologia para o Instituto, resgatando o papel de avaliador externo do Sistema Único de Saúde (SUS).

Palavras-chave Inquérito domiciliar, PNAD COVID-19, Brasil 


\section{Introdução}

Até o dia $1^{\circ}$ de julho de 2020, o Brasil registrou o segundo maior número de casos confirmados da COVID-19 (1,4 milhões) no mundo, com uma taxa de mortalidade de 269/milhão de habitantes, com 14.445 testes por milhão de habitantes ${ }^{1}$, ficando apenas atrás do Uruguai com 19.086 testes por milhão, Colômbia (14.612/1 milhão), Argentina (7.798/1 milhão) e Paraguai (9.723/1 milhão). A taxa de letalidade em nosso país foi de $4,2 \%$, com a nota de que ela deve ser, de fato, mais baixa ao admitir-se que, pelo menos, $40 \%$ dos casos são assintomáticos ${ }^{2}$ e, portanto, devem configurar no denominador do indicador ao se calcular essa taxa.

De forma inédita, o IBGE utilizou na PNAD-COVID-19 o parâmetro de semana epidemiológica, e dessa forma, divulgou em junho de 2020 os primeiros resultados como casos referidos nas $19^{\mathrm{a}}$ a $22^{\mathrm{a}}$ semanas epidemiológicas de ocorrência ${ }^{3}$. É o primeiro Instituto Nacional de Estatística de um país no mundo inteiro a realizar esse tipo de levantamento amostral de base domiciliar, com coleta de dados por entrevistas telefônicas durante a pandemia de SARS-CoV-2. O inquérito COVID- $19^{4}$ foi composto por um pequeno módulo de sete perguntas dentro da Pesquisa Nacional por Amostra de Domicílios Contínua (PNAD-C), que teve como principal objetivo quantificar os principais efeitos da pandemia no mercado de trabalho.

\section{A PNAD COVID-19}

Na PNAD COVID-19, realizada em parceria com o Ministério da Saúde, os dados coletados tiveram como referência a população-residente brasileira estimada em 210,1 milhões de habitantes. Em maio/2020, a amostra pesquisou 349 mil pessoas referenciadas a cerca de 200 mil domicílios. $\mathrm{O}$ morador que atendesse ao telefone respondia o questionário de coleta de dados por todos os moradores do domicílio. As amostras probabilísticas foram desenhadas para permitir estimativas representativas para cada uma das 27 unidades da federação, e as cinco macrorregiões geográficas do país.

O módulo intitulado "COVID-19" abordou questões relacionadas aos 12 sintomas de síndrome gripal. A pergunta principal do modulo referia-se à presença/ausência desse sinais e sintomas na semana anterior ("Na semana passada, o(a) $\mathrm{Sr}(\mathrm{a})$ teve: [febre, tosse, dor de garganta, dificul- dade para respirar, dor de cabeça, dor no peito, náusea, nariz entupido ou escorrendo, fadiga, dor nos olhos, perda de cheiro ou de sabor, dor muscular]").

O módulo COVID-19 é complementado por questões que detalham outros aspectos das pessoas com sintomas referidos, trazendo perguntas relacionadas à procura de um estabelecimento de saúde (inclusive as unidades de atenção primária à saúde e as equipes de saúde da família); providências tomadas para se recuperar dos sintomas; local em que buscou atendimento; necessidade de internação/ sedação/ intubação e respiração artificial. Essas perguntas podiam ser ainda associadas a outro modulo da PNAD que caracterizava cada morador ou domicílio: sexo, faixa etária, cor ou raça, condição no domicílio, escolaridade, posse de plano de saúde. Por exemplo, análise dos microdados da pesquisa demonstrou que quanto maior a escolaridade, maior a proporção de pessoas com sintomas de síndrome gripal.

Do total da população-residente brasileira, o IBGE estimou em maio/2020 que 24,0 milhões $(11,4 \%)$ tiveram pelo menos um dos sintomas de síndrome gripal (SG). Quando se desagrega esse dado entre os domicílios com apenas um morador (arranjos unifamiliares) x domicílios com mais de um morador (arranjos multifamiliares), os resultados em termos absolutos são muito próximos, isto é, no primeiro caso, 10,7 milhões apresentaram algum sintoma de SG, enquanto 13,3 milhões nos arranjos multifamiliares.

Dos 24 milhões de brasileiros que apresentaram algum sintoma de síndrome gripal no mês de maio/2020, 20,2 milhões (84,3\% do total dos sintomáticos) não procuraram unidade de saúde por diversos motivos. Dentre esses, 16,8 milhões $(82,9 \%)$ ficaram em casa, 1,2 milhão $(5,8 \%)$ ligaram para algum profissional de saúde, 11,5 milhões $(56,9 \%)$ compraram ou tomaram remédio por conta própria, 2,9 milhões $(14,5 \%)$ compraram ou tomaram remédio por orientação médica, 173 mil $(0,9 \%)$ receberam visita de profissional de saúde particular e 526 mil (2,6\%) receberam visita de algum profissional de saúde da atenção primária à saúde do SUS. Entre esses moradores que receberam visitas das Equipes de Saúde da Família, 20\% residiam na região Norte, $43,7 \%$ no Nordeste, $20,6 \%$ no Sudeste, $10,8 \%$ no Sul e $5,0 \%$ no Centro-Oeste.

Portanto, com esses dados, poder-se-ia levantar uma hipótese que a taxa de letalidade poderia ser mais baixa se somássemos o total de pessoas com sintomas apontadas pela PNAD COVID-19, que não foram confirmadas laboratorialmente 


\section{Inovações trazidas pela PNAD COVID-19 - IBGE}

A informação é fundamental para o controle de qualquer doença, mas ela se torna especialmente importante em se tratando de uma doença transmissível nova, sem medicamentos ou vacinas eficazes disponíveis, em que as medidas de controle dependem tão intrinsicamente do conhecimento epidemiológico. A vigilância em saúde é a responsável por coletar sistematicamente as informações relevantes para a saúde da população, usando tradicionalmente notificações de casos que se apresentam em unidades de saúde e laboratórios como sua fonte primária de dados. Como alternativa a dados gerados durante a assistência à saúde, iniciativas como inquéritos sorológicos, vigilância participativa e até atividade online relacionada a certas palavras-chaves têm sido utilizadas. Neste contexto, a PNAD COVID-19 trouxe um conjunto de inovações para a vigilância em saúde.

Em primeiro lugar ela se apresenta como uma ferramenta robusta na busca por informações de saúde. Sua robustez está relacionada à associação à PNAD-C, instrumento de validade estabelecida com amostra representativa para todos os estados da federação. Iniciativas de vigilância ativa em saúde habitualmente carecem desse lastro estatístico que a experiência e a $e x$ pertise única do IBGE conferem à metodologia, que estima inclusive os coeficientes de variação para cada estatística apurada.

Em segundo lugar, ela busca por sinais e sintomas. A PNAD COVID-19 coleta 12 sinais e sintomas possivelmente associados à COVID-19. Ao invés de buscar diagnósticos ou fatores de risco, trata-se de um inquérito de vigilância sindrômica. Seus resultados trazem informações diferentes das que estavam disponíveis até junho de 2020, sendo fundamental entender como utilizá-las da melhor forma. Uma das limitações da maior parte das fontes de informação está em restringir suas definições de caso a um pequeno número de manifestações clínicas. No caso das doenças infecciosas agudas de maneira geral, e, particularmente no caso de novas doenças emergentes ainda sendo caracterizadas clinicamente, utilizar três ou quatro sintomas para classificar casos suspeitos - e até mesmo para definir quem será testado para confirmação laboratorial - exclui dos números oficiais um contingente enorme de casos com apresentação clínica às vezes nem tão atípicas. Mesmo estudos que buscam fatores clínicos preditores de diagnóstico sofrem com o viés de inclusão. A possibilidade de utilizar diferentes combinações de sinais e sintomas como critérios de definição, reconhecendo que apresentarão diferentes sensibilidade e especificidade, aumentam sua flexibilidade e tornam os dados úteis para diferentes contextos e objetivos de análise.

Em terceiro lugar, ela traça uma perspectiva longitudinal. A PNAD COVID-19 coleta dados semanalmente, tendo como referência o mesmo conceito de semana epidemiológica utilizada pela vigilância em saúde, permitindo um acompanhamento seriado único dos domicílios brasileiros, característica que a distingue de outros inquéritos transversais com amostras independentes.

Em quarto lugar, ela realiza um levantamento de dados peculiar quanto ao desfecho. Ao se desvincular da coleta de dados em unidades de saúde, o inquérito traz dados que não seriam coletados pelos mecanismos tradicionais da vigilância em saúde. O desenho da PNAD COVID-19 permite coletar informações sobre oligossintomáticos, importantes na cadeia de transmissão. Rompe também a barreira de acesso aos serviços de saúde, que é uma barreira não só para a atenção à saúde, mas também para a coleta de dados pela vigilância.

Em quinto lugar, ela permite analisar a integração entre atenção primária e vigilância à saúde, fundamental em tempos de pandemia, pois como é conhecido desde o primeiro estudo publicado que classificou os sintomas da COVID- $19^{10}$, onde $80,9 \%$ das pessoas que tiveram a doença apresentam sintomas leves, e, portanto, não necessitariam de internação. Por isso, o papel da atenção primária e sua integração junto à vigilância em saúde tem sido destacado em alguns países como Portugal que desde o início abriu "áreas dedicadas à COVID-19" (ADC) coordenadas pelos cuidados de saúde primários ${ }^{11}$, tendo sido essa iniciativa apontada como uma das razões para o sucesso do combate ao COVID-19 naquele país, para além das testagem em massa 
(mais de 10\% da população-residente), do isolamento social e do uso de equipamentos de proteção individual pela população.

A PNAD COVID-19 destacou que entre aqueles que tiveram sintomas de síndrome gripal e buscaram um estabelecimento de saúde, a maior parte das pessoas foram a uma unidade de atenção primária e procuraram um profissional de uma Equipe de Saúde da Família (eSF). Se não fossem as ações desenvolvidas nos territórios pelas eSF, a procura pelos serviços de pronto-atendimento e hospitalares teria sido muito maior. Em Portugal, por exemplo, essas ações das equipes de atenção primária evitaram o colapso nas unidades hospitalares.

Em sexto lugar, o inquérito permite associar os dados dos brasileiros acima de 14 anos com sintomas de síndrome gripal aos dados de mercado de trabalho, também coletados, para medir os efeitos da pandemia nessa área.

Finalmente, seu desenho em coletar dados usando semana epidemiológica permite a comparação com os dados informados no e-SUS e no SIVEP-Gripe.

\section{Colaboradores}

GO Penna, J Cerbino Neto e LF Pinto fizeram o levantamento de dados e a redação da primeira versão do texto. JAA Silva e JG Temporão participaram das versões consecutivas e revisando-as criticamente.

\section{Considerações finais}

Os dados de casos novos confirmados, tanto por inquéritos sorológicos quanto pela assistência à saúde, devem ser usados para encontrar a correspondência entre os dados da PNAD COVID-19 e a circulação do SARS-CoV-2 no país e em cada UF, uma vez que outros vírus respiratórios irão causar sintomas semelhantes. A análise dessas informações em conjunto é que permitirá extrair as principais conclusões epidemiológicas da PNAD COVID-19.

O pioneirismo do IBGE demonstra ser possível, em um país continental e que vem experimentando diversas epidemias locais em momentos diferentes em seu território, que outros países também desenvolvam inquéritos domiciliares semelhantes, com coleta de dados por telefone de forma inovadora e tempestiva. A PNAD COVID-19 traz ainda uma nova tecnologia para o Instituto, resgatando o papel de avaliador externo do Sistema Único de Saúde (SUS). 


\section{Referências}

1. Worldometer. COVID-19 Coronavirus Pandemic [Internet]. [acessado 2020 Jun 20]. Disponível em: https://www.worldometers.info/coronavirus/

2. Lavezzo E, Franchin E, Ciavarella C, Cuomo-Dannenburg G, Barzon L, Del Vecchio C, Rossi L, Manganelli R, Loregian A, Navarin N, Abate D, Sciro M, Merigliano S, De Canale E, Vanuzzo MC, Besutti V, Saluzzo F, Onelia F, Pacenti M, Parisi S, Carreta G, Donato D, Flor L, Cocchio S, Masi G, Sperduti A, Cattarino L, Salvador R, Nicoletti M, Caldart F, Castelli G, Nieddu E, Labella B, Fava L, Drigo M, Gaythorpe KAM, Imperial College COVID-19 Response Team, Brazzale AR, Toppo S, Trevisan M, Baldo V, Donnelly CA, Ferguson NM, Dorigatti I, Crisanti A. Suppression of a SARS-CoV-2 outbreak in the Italian municipality of Vo'. Nature 2020 [preprint]. [acessado $2020 \mathrm{Jul}$ 04]. Disponível em: https://www.nature.com/articles/ s41586-020-2488-1

3. Brasil. Instituto Brasileiro de Geografia e Estatística (IBGE). Pesquisa Nacional por Amostra de Domicílios Contínua (PNAD) COVID-19. Microdados [Internet]. Rio de Janeiro: IBGE; 2020 [acessado 2020 Jun 24]. Disponível em: ftp://ftp.ibge.gov.br/Trabalho_e_Rendimento/Pesquisa_Nacional_por_Amostra_de Domicilios_PNAD_COVID19/Microdados/PNAD COVID19_052020.zip

4. Brasil. Instituto Brasileiro de Geografia e Estatística (IBGE). Instrumentos de coleta. PNAD COVID-19 [Internet]. [acessado 2020 Jun 20]. Disponível em: https://biblioteca.ibge.gov.br/visualizacao/instrumentos_de_coleta/doc5586.pdf

5. Wu Z, McGoogan JM. Characteristics of and important lessons from the coronavirus disease 2019(COVID-19) outbreak in China: summary of a report of 72314 cases from the Chinese Center for DiseaseControl and Prevention. JAMA 2020; 323(13):1239-1242.

6. Lapostolle F, Schneider E, Vianu I, Dollet G, Roche B, Berdah J, Michel J, Goix L, Chanzy E, Petrovic T, Adnet F. Clinical features of 1487 COVID-19 patients with outpatient management in the Greater Paris: the COVID-call study. Intern Emerg Med 2020; 1-5.
7. Menni C, Valdes AM, Freidin MB, Sudre CH, Nguyen LH, Drew DA, Ganesh S, Varsavsky T, Cardoso MJ, Moustafa JSE-S, Visconti A, Hysi P, Bowyer RCE, Mangino M, Falchi M, Wolf J, Ourselin S, Chan AT, Stevens CJ, Spector TD. Real-time tracking of self-reported symptoms to predict potential COVID-19. Nature Med 2020; 1-8.

8. Dados do Bem [Internet]. [acessado 2020 Jun 24]. Disponível em: https://dadosdobem.com.br/

9. British Medical Journal (BMJ) Best Practice. Coronavirus disease 2019 (COVID-19) [Internet]. [acessado 2020 Jun 20]. Disponível em: https://bestpractice.bmj. com/topics/en-gb/3000168/history-exam

10. Team The Novel Coronavirus Pneumonia Emergency Response Epidemiology (NCPERE). Vital Surveillances: The Epidemiological Characteristics of an Outbreak of 2019 Novel Coronavirus Diseases (COVID-19) - China, 2020. China CDC Weekly 2020; 2(8):113-22.

11. Portugal. ARSLVT abre 35 Áreas Dedicadas à COVID-19 (ADC) nos Cuidados Saúde Primários [Internet]. Lisboa: ARSLVT; 2020. [acessado 2020 Jun 28]. Disponível em: https://www.arslvt.min-saude.pt/ frontoffice/pages $/ 2$ ?news_id $=2396$

Artigo apresentado em 04/07/2020

Aprovado em 05/07/2020

Versão final apresentada em 07/07/2020 
